\title{
Characterisation of human iPS cells harbouring the p.A329T variant of caspase-1
}

\author{
J Thiem* , A Rösen-Wolff \\ From 8th International Congress of Familial Mediterranean Fever and Systemic Autoinflammatory Diseases \\ Dresden, Germany. 30 September - 3 October 2015
}

\section{Introduction}

Naturally occurring genetic variants of the CASP1 gene are associated with autoinflammation in patients suffering from recurrent febrile episodes and generalized inflammation. The resulting caspase- 1 variants have reduced enzymatic activity due to destabilization of the caspase-1 tetramer. In addition, autoprocessing of the pro-caspase- 1 variants is reduced and hence, CARDCARD interactions with RIP2, enabling NFkB activation, is enhanced in a cell culture model.

\section{Objectives}

In order to characterize the effects of the p.A329T caspase1 variant detected in a child suffering from severe autoinflammatory symptoms, we tried to establish a reliable cell model based on patient derived induced pluripotent stem cells (iPSCs).

\section{Methods}

We generated iPSCs by reprogramming fibroblasts of the patient's tympanic membrane (and wild type fibroblasts) using a replication deficient retrovirus (pRRL.PPT.SF. hOKSMco.idTomato.preFRT pMD.G (VSVG) psPAX2). We were able to verify the pluripotency of these iPSCs in different tests (quantitative real time PCR analysis of pluripotency markers, Immunocytochemistry of three germ layer markers and pluripotency markers). Subsequently the resulting iPSCs formed embryoid bodies (EB) which were then cultured in X-Vivo 15 media containing M-CSF and IL-3. After a few weeks, the adherent cells spreading from the settled EBs began to release monocytes (CD14+, CD45+, CD105+, CD192+). These were harvested from the supernatant and differentiated for 7 days in the presence of M-CSF (without IL-3). Thereafter, they were detached, counted and plated as $1 \times 10^{5}$ cells per 96 -well.
After allowing them to rest for one day, we primed the cells with ultrapure LPS and stimulated them with ATP or Nigericin. The supernatant was analyzed for cytokine concentrations of IL-1 $\beta$, IL-6, IL-10 and TNF $\alpha$ using BD ${ }^{\mathrm{TM}}$ Cytometric Bead Array.

\section{Results}

The generated monocytes expressed the commonly established cell surface markers (CD14, CD16, CD45, CD163, and CD192) and could be differentiated into functional macrophages that seemed to react to stimulation as expected. Furthermore, first results indicated a different reaction of the cells generated from the patient (p.A329T) in comparison to the wild type control.

\section{Conclusion}

Human induced pluripotent stem cells are a useful option to gain monocytes and macrophages in order to study defects of the immune system without the necessity to repeatedly draw large amounts of blood.

\section{Acknowledgements}

This study was supported by German Network on Primary Immunodeficiency Diseases (pid.net).

Published: 28 September 2015

doi:10.1186/1546-0096-13-S1-P33

Cite this article as: Thiem and Rösen-Wolff: Characterisation of human iPS cells harbouring the p.A329T variant of caspase-1. Pediatric Rheumatology 2015 13(Suppl 1):P33. 